\title{
A Subspace Approach for Approximation of Rational Matrix Functions to Sampled Data ${ }^{1}$
}

\author{
Tomas McKelvey and Anders Helmersson \\ Dept. of Electrical Engineering, Linköping University \\ S-581 83 Linköping, Sweden, \\ Email: tomas@isy.liu.se, andersh@isy.liu.se. \\ CDC 1996, Kobe Japan
}

\begin{abstract}
Algorithms for approximation of rational matrix factors to data is described. The method is based on a subspace based multivariable frequency domain statespace identification, canonical and spectral factorization and parametric optimization. The algorithms can be used for identifying spectral factors and factors of positive real functions from frequency data. The methods are directly applicable in the $D-K$ algorithm for complex $\mu$-synthesis and the $Y-Z-K$ algorithm for mixed $\mu$-synthesis.
\end{abstract}

\section{Notation}

$\mathcal{R} \mathcal{L}_{\infty}$ is the set of real-rational matrix functions with no poles on the imaginary axis. Let $\mathcal{R} \mathcal{H}_{\infty}=\{X(s)$ : $X(s) \in \mathcal{R L}_{\infty}, X(s)$ analytic in $\left.\operatorname{Re}(s)>0\right\}$ be the set of stable real-rational matrix functions. Let $A^{*}$ denote the conjugate transpose of $A$ and let $\|\cdot\|$ denote any matrix norm and $G^{\sim}(s)=G^{T}(-s)$.

\section{Introduction}

Realization and approximation of matrix functions plays an important role in many automatic control and signal processing applications. In this paper subspace based approximations are proposed and discussed. The methods presented are focused on state-space realizations of scalings and multipliers in connection with $D$ $K$ iterations (spectral factorization) $[2,3]$ and $Y-Z$ $K$ iterations [6, 5] used for robust controller design. The method proposed in this paper is also applicable when finding a rational spectral factor from a measured power spectrum of a multivariable disturbance. For optimal filtering such a factor of the disturbance spectrum is needed [1].

\footnotetext{
${ }^{1}$ This work was supported in part by the Swedish Research Council for Engineering Sciences (TFR), which is gratefully acknowledged.
}

The problem treated is to find a state-space realization which approximates given multivariable frequency data $W_{k} \in \mathbb{C}^{p \times p}$ sampled at frequencies $\omega_{k}$.

In the spectral factorization problem $W_{k}=W_{k}^{*}>0$ and a spectral factor $\hat{G}(s)$ is sought which minimizes

$$
\sum_{k}\left\|W_{k}-\hat{G}\left(j \omega_{k}\right)^{*} \hat{G}\left(j \omega_{k}\right)\right\|^{2}
$$

where $\hat{G}, \hat{G}^{-1} \in \mathcal{R} \mathcal{H}_{\infty}$, i.e. $\hat{G}$ is a stable and inversely stable real rational matrix.

The second problem which arises in the $Y-Z-K$ algorithm deals with positive real data $W_{k}$, i.e. $W_{k}+$ $W_{k}^{*}>0$ and two factors $\hat{Y}(s)$ and $\hat{Z}(s)$ are sought such that

$$
\sum_{k}\left\|W_{k}-\hat{Y}\left(j \omega_{k}\right)^{*} \hat{Z}\left(j \omega_{k}\right)\right\|^{2}
$$

is minimized and $\hat{Y}, \hat{Y}^{-1}, \hat{Z}, \hat{Z}^{-1} \in \mathcal{R} \mathcal{H}_{\infty}$.

By parametrizing the unknown factors $(G, Y, Z)$ a nonlinear constrained parametric optimization problem results which have to be solved by iterative methods. In order for such a method to be successful for any nontrivial case, high quality initial estimates of the unknown factors have to be determined. This paper will focus on algorithms for deriving these initial estimates.

\section{Preliminaries}

First we revise some known results on factorization of square rational matrices.

Lemma 1 (Spectral factorization [4]) Assume that $W(s)=W^{\sim}(s)>0, W(\infty)>0$ and $W, W^{-1} \in$ $\mathcal{R} \mathcal{L}_{\infty}$. Then there exist matrix functions $G$ such that $W(s)=G^{\sim}(s) G(s)$ and $G, G^{-1} \in \mathcal{R H}_{\infty}$.

Lemma 2 (Positive real factorization [6, 4]) Assume that $W(j \omega)+W^{\sim}(j \omega)>0, \forall \omega \in \mathbb{R} \cup\{\infty\}$ 
and $W, W^{-1} \in \mathcal{R} \mathcal{L}_{\infty}$. Then there exist matrix functions $Y, Z$, such that $W(s)=Y^{\sim}(s) Z(s)$ and $Y, Y^{-1}, Z, Z^{-1} \in \mathcal{R} \mathcal{H}_{\infty}$.

Constructive state-space algorithms exist for these factorizations, see $[4,8]$.

\section{Basic Algorithm}

The solution to the approximation problem can be split into three main steps

Step 1 Approximation of a rational matrix $\hat{W}(s) \in$ $\mathcal{R} \mathcal{L}_{\infty}$ such that $\sum_{k}\left\|W_{k}-W\left(j \omega_{k}\right)\right\|^{2}$ is small. This is done with a subspace based frequency domain state-space identification algorithm [7]. In this first approximation step we impose no restrictions on $\hat{W}(s)$.

Step 2 First it is checked if $\hat{W}(s)+\hat{W}(s)^{*}>0$. If not a modification $\gamma>0$ is introduced

$$
\hat{\hat{W}}(s):=\hat{W}(s)+\gamma I
$$

such that $\hat{\hat{W}}(j \omega)+\hat{\hat{W}}(j \omega)^{*}>0$. To find a suitable $\gamma$ is a convex problem which can be solved by an LMI using the Kalman-Yakubovich-Popov lemma or by a simple bisection technique checking the eigenvalues of the associated Hamiltonian matrix.

A factorization $\hat{\hat{W}}=\hat{Y}^{\sim} \hat{Z}$ according to Lemma 2 is then well defined.

Step 3 The obtained factors are converted to some state-space basis suitable for parametrization and the iterative parametric optimization of (1) or (2) can be performed.

This basic algorithm can directly be used to the problem given in equation (2). The spectral factorization problem can be solved using some variations of this basic algorithm. If modifications are necessary in step 2 $(\gamma>0)$ the quality of the approximation obtained by the subspace method in step 1 becomes degraded and step 3 is instrumental in order to obtain good results.

\section{Spectral Factorization}

Since Step 1 in the algorithm finds a rational approximation to the given data without imposing any constraints it is most likely that the obtained approximation does not satisfy $\hat{W}=\hat{W}^{\sim}$, and consequently Lemma 1 cannot be applied. Two possibilities immediately emerges: 1) Use Lemma 2 to obtain a factorization $\hat{W}=\hat{Y}^{\sim} \hat{Z}$ and let $\hat{G}:=\hat{Z}$ be the spectral factor.
However for some data it can happen that the orders of $Y$ and $Z$ are not equal which gives an indication of a potentially bad approximation. A second approach can then be applied: 2) Let $\hat{\hat{W}}:=\frac{1}{2}\left(\hat{W}+\hat{W}^{\sim}\right)$ which is Hermitian and Lemma 1 applies. For computational reasons it is better to use the result in [8, Theorem 13.25] which directly uses $\hat{W}$ and never forms $\hat{\hat{W}}$. The drawback of this second approach is that the order of the factor $\hat{G}$ is doubled. Prior to the optimization we recommend to reduce the order by a balanced truncation. The truncation preserves the stability of $\hat{G}$. Inverse-stability of the reduced factor can be recovered, if necessary, by a second spectral factorization.

\section{Conclusions}

This paper presents algorithms for realization of statespace representations of matrix functions given as frequency sampled data. The algorithm is based on subspace identification. The method is geared towards solving realization problem in $D-K$ and $Y-Z-K$ iterations when designing robust controllers.

The subspace method combined with canonical factorization show promising results for $D-K$ iterations. It is general in the respect that it can be used for multivariable scaling as well as real- $\mu$ synthesis.

\section{References}

[1] B. D. O. Anderson and J. B. Moore. Optimal Filtering. Prentice-Hall, Englewood Cliffs, New Jersey, 1979.

[2] J. C. Doyle. Structured uncertainty in control systems design. In Proc. of the 24th IEEE Conference on Decision and Control, pages 260-265, Fort Lauderdale, Florida, December 1985.

[3] J. C. Doyle, K. Lentz, and A. Packard. Design examples using $\mu$-synthesis: Space shuttle lateral axis FCS during reentry. In IEEE Proceedings of the 25th Conference on Decision and Control, volume 3, pages 2218-2223, Athens, Greece, December 1986.

[4] B. Francis. A Course in $H_{\infty}$ Control Theory, volume 88 of Lecture Notes in Control and Information Science. New York: Springer Verlag, 1987.

[5] A. Helmersson. Applications of mixed- $\mu$ synthesis using the passivity approach. In Proceedings of the 3rd European Control Conference, volume 1, pages 165-170, Rome, Italy, September 1995.

[6] A. Helmersson. Methods for Robust Gain Scheduling. $\mathrm{PhD}$ thesis, Linköpings universitet, Linköping, Sweden, 1995.

[7] T. McKelvey, H. Akçay, and L. Ljung. Subspacebased multivariable system identification from fre- 
quency response data. IEEE Trans. on Automatic Control, 41(7):960-979, July 1996.

[8] K. Zhou, J. C. Doyle, and K. Glover. Robust and Optimal Control. Prentice Hall, Upper Saddle River, NJ, 1996. 